\author{
ACTA MYCOLOGICA \\ Vol. 43 (1): 105-111 \\ 2008
}

\title{
Lichens of the Holy Hill orthodox sanctuary in Grabarka (NE Poland)
}

\author{
ANNA MATWIEJUK \\ Department of Botany, Institute of Biology, University of Bialystok \\ Świerkowa 20B, PL-15-950 Białystok, matwiej@uwb.edu.pl
}

Matwiejuk A.: Lichens of the Holy Hill orthodox sanctuary in Grabarka (NE Poland). Acta Mycol. 43 (1): 105-111, 2008.

The Holy Hill Grabarka is one of the most important orthodox sanctuaries in Poland. The sanctuary is situated in Podlasie region between Bug and Narew rivers. It grew in the shade of well developing (in the first centuries of the second millennium) towns Mielnik and Drohiczyn. The most striking thing is that the church is surrounded by a forest of thousands of crosses brought by pilgrims. The study present 64 species of epiphytic, epixylic, epilythic and epigeic lichens. Among 64 lichen species 11 are considered to be threatened in Poland.

Key words: lichenized fungi, biota, Holy Hill sanctuary

\section{INTRODUCTION}

The Holy Hill Grabarka is the most significant Orthodox sanctuary in Poland. The sacred place is situated $12 \mathrm{~km}$ away from Siemiatycze, $0,5 \mathrm{~km}$ from the Grabarka village in Podlaskie province, Siemiatyckie district, commune Nurzec-Stacja. The Holy Hill is found on Wysoczyzna Drohicka High Plain, in the south part of Nizina Północnopodlaska Lowland (Kondracki 1994). In the close neighborhood there is the river Moszczona, which falls into the river Bug, $7 \mathrm{~km}$ further to the south. The surrounding woods had been formerly included in the Mielnicka Forest, which is known as the Lasy Nurzeckie Forests.

\section{STUDY AREA}

The Holy Hill Grabarka acquired its fame for a miracle which occurred in the beginning of 1710 in the time of cholera epidemic, which decimated the population of Podlasie. As legend says, at that time, an old man from Siemiatycze had a revelation according to which it was possible to be protected from the disease and be saved only on the Grabarka Hill. After the faithful from Siemiatycze had arrived and placed there a cross, a miracle occurred. The sick started to recover and nobody died afterwards. When the news spread, people from that region arrived at the Holy Hill 
in hundreds. In 1710, in summer, about 10 thousand people gathered in this place. In the same year, a wooden temple was erected there as gratitude of those who were saved. The Holy Grabarka Hill is also known as the Hill of Crosses- the name comes from sacrificial crosses with epitaphs written in Cyrillic alphabet, which are placed around the Transfiguration Orthodox Church. The crosses which surround the small church have become the symbol of the Holy Hill. It is a real forest of crosses with narrow paths to walk along (Radziukiewicz 2001).

At present, approximately 10 thousand crosses tower on the hill - wooden, metal, stone, small and big. The crosses have been traditionally brought by the faithful for hundreds of years. The pilgrims place them next to the sanctuary throughout the year, however the most crosses occur the day before the Transfiguration Holiday (Lechowski 2004).

Behind the amazing forest of crosses, a cemetery and buildings of the St. Martha and St. Mary are found. The nunnery was established in 1947 and the Holy Virgin Patroness and Succor Orthodox church was erected in 1957. Construction of stone wall to enclose the Holy Hill was begun in 1999 as a result of benediction of His Eminence Metropolitan Sawa (Radziukiewicz 2000).

The objective of the present paper has been the floristic and ecological analysis of lichen biota of the Holy Grabarka Hill and its surrounding for the purpose of presenting its variety considering the habitat conditioning.

\section{MATERIAL AND METHODS}

The investigations in the area of the Holy Hill orthodox sanctuary in Grabarka and its surroundings were carried out in the years 2006-2007. The alphabetical list of lichen species has been compiled (Tab. 1). Several lichen species from the Grabarka surroundings (stand 1182 - Alnus glutinosa over flow, sandy slope, Pinus sylvestris) have been reported by Cieśliński (2003a). The species confirmed by our own studies on that stand have been marked (also Cieśliński 2003a). The species which have not been identified in the course of our own studies have been marked Cieśliński (2003 a). The species have been named according to Santesson et. al. (2004), genera Bryoria and Usnea according to Bystrek $(1986,1994)$ and genus Melanelia according to Blancoet al. (2004).

The lichen material has been deposited at the Herbarium of the Institute of Biology, University of Białystok.

\section{RESULTS}

64 species of lichens, out of 35 genera have been identified on the area of the Holy Grabarka Hill and its neighborhood. The most abundant are genera Cladonia -8 species, Lecanora - 7, Caloplaca - 4. The lichens occur in all basic morphological forms. Crustose thalus species remarkably dominate (45\%). There are less macrolichens - foliose (29\%) and fruticose (25\%). Of the 64 lichen species identified in the Holy Hill orthodox sanctuary in Grabarka and its environs, 11 species have been put on the Red List of extinct and vulnerable lichens of Poland (Cieśliński et al. 2003), including 3 species in the endangered category - EN (Bryoria crispa, Flavoparmelia caperata, Pleurosticta acetabulum), 6 species in the vulnerable category - VU 
(Cetraria islandica, Parmelina tiliacea, Porpidia rugosa, Tuckermanopsis chlorophylla, Usnea filipendula, U. hirta), 2 species in the category of near threatened - NT (Evernia prunastri, Hypogymnia tubulosa), as well as 2 species on the Red List of lichens vulnerable in North-Eastern Poland (Cieśliński 2003 b), including 1 species in the category EN (Flavoparmelia caperata), 1 - LC (Porpidia rugosa).

Lichens which are protected by law constitute $27 \%$ of the total lichen biota, including strict environmental protection species $-20 \%$, and partial protection $-6 \%$. Zones of protection within a radius of $50 \mathrm{~m}$ from the rim of the species locality should be specified in regard to Usnea hirta and Usnea filipendula (Dz. U. Nr 168, poz. 1765).

Epiphytic lichens. The most abundant are epiphytic lichens (31 species), out of which 11 are obligatory. They occur on the bark of Pinus sylvestris, Betula pendula, Quercus robur, Acer platanoides, Alnus glutinosa and Juniperus communis. Considering floristic differentiation and abundance, the richest is lichen biota Pinus sylvestris (11 species), Acer platanoides and Betula pendula (10 of each). Fruticose species, generally perishing in Poland - Usnea hirta, U. filipendula, Pseudeuernia furfuracea as well as foliose Hypogymnia physodes occur in considerable amount on the bark of pine - and birch - trees. The lower parts of the trunks are mainly colonized by the lichens of Cladonia genus (C. chlorophaea, C. coniocraea, $C$. fimbriata). The bark of numerous trunks, including the crown, is profusely overgrown with lichens thalus. 11 species, exclusively Flavoparmelia caperata (Cieśliński 2003a), Lecanora carpinea, L. pulicaris, Pleurostica acetabulum (Cieśliński 2003b) were identified on the bark of alders growing on the stream. Species composition of the lichens of pine - trees growing in the nearest woods is remarkabely scantier. Common species such as Hypogymnia physodes, Hypocenomyce scalaris, Cladonia coniocraea significantly dominate, and Imshaugia aleurites and Lecanora conizaeoides are found relatively often.

Epixylic lichens. These lichens are included in the second, regarding the number, habitat group. They are represented by 24 species, out of which only 3 (Lecanora varia, Parmelina tiliacea, Placynthiella icmalea) are exclusive epixylic lichens. They mainly grow on the wooden crosses which surround the church. Quite frequently they form big groups composed of a dozen or so - several dozens specimens. Mainly epiphytic species occur among epixylic species. The most abundant are foliose thalus Platisimatia glauca, Parmelia sulcata, Hypogymnia physodes, Parmelina tiliacea, Melanelixia fuliginosa, Imshaugia aleurites as well as fruticose thalus Pseudevernia furfuracea, Evernia prunastri, Usnea hirta, U. filipendula, Cladonia fimbriata, C. coniocraea. Wood left to rot after the cut down trees form the second breeding ground overgrown by epixylic lichens, such as Hypogymnia physodes or Imshaugia aleurites, Lepraria sp., Parmelia sulcata, Placynthiella icmalea.

Epilithic lichens. 24 species have been identified, out of which 19 exclusive. Lichens of the Holy Grabarka Hill are connected with natural breeding ground (erratic blocks, stones, tombstones, stone wall) - 15 species, out of which Caloplaca holocarpa, Candelariella aurella, Lecanora albescens, L. dispersa, Physcia caesia, Protoparmeliopsis muralis, Xanthoria parietina and $X$. polycarpa grow also on calcium breeding ground, on concrete structures mainly (tombstones). 9 species were identified exclusively on breeding ground of calcium substrate. In the Grabaraka vicinity, Cieśliński (2003a) identified Porpidia rugosa (individual without apothecium) on 
Table 1

Lichens recorded on the Holy Hill in Grabarka

\begin{tabular}{|c|c|c|}
\hline SPECIES & SUBSTRATUM & NOTES \\
\hline Acarospora fuscata (Schrad.) Th. Fr. & erratic blocks, stone wall & also Cieśliński (2003a) \\
\hline $\begin{array}{l}\text { Amandinea punctata (Hoffm.) Coppins \& } \\
\text { Scheid. }\end{array}$ & trunk of Acer platanoides & \\
\hline Bryoria crispa (Mot.) Bystr. & trunk of Pinus sylvestris & \\
\hline Caloplaca citrina (Hoffm.) Th. Fr. & concrete tombstones & \\
\hline $\begin{array}{l}\text { Caloplaca decipiens (Arnold) Blomb. \& } \\
\text { Forssell }\end{array}$ & concrete tombstones & \\
\hline $\begin{array}{l}\text { Caloplaca holocarpa (Hoffm. ex Ach.) A. E. } \\
\text { Wade }\end{array}$ & $\begin{array}{l}\text { stones, stone wall, concrete } \\
\text { tombstones }\end{array}$ & \\
\hline Caloplaca saxicola (Hoffm.) Nordin & concrete tombstones & \\
\hline Candelariella aurella (Hoffm.) Zahlbr. & $\begin{array}{l}\text { concrete tombstones, stone } \\
\text { wall }\end{array}$ & \\
\hline Candelariella vitellina (Hoffm.) Müll. Arg. & concrete tombstones & \\
\hline Candelariella xanthostigma (Ach.) Lettau & trunk of Acer platanoides & \\
\hline Cetraria aculeata (Schreb.) Fr. & soil & also Cieśliński (2003a) \\
\hline Cetraria islandica (L.) Ach. & soil & also Cieśliński (2003a) \\
\hline $\begin{array}{l}\begin{array}{l}\text { ladonia arbuscula (Wallr.) Flot. ssp. mitis } \\
\text { (Sandst.) Ruoss }\end{array} \\
\end{array}$ & soil & also Cieśliński (2003a) \\
\hline $\begin{array}{l}\text { Cladonia chlorophaea (Flörke ex Sommerf.) } \\
\text { Spreng. }\end{array}$ & soil & also Cieśliński (2003a) \\
\hline $\begin{array}{l}\text { Cladonia coniocraea (Flörke) Spreng., nom } \\
\text { cons. }\end{array}$ & $\begin{array}{l}\text { soil, trunk of Pinus sylvestris, } \\
\text { Betula pendula, wooden } \\
\text { crosses, decaying wood }\end{array}$ & also Cieśliński (2003a) \\
\hline Cladonia fimbriata (L.) Fr. & $\begin{array}{l}\text { soil, trunk of Pinus sylvestris, } \\
\text { wooden crosses, decaying } \\
\text { wood }\end{array}$ & also Cieśliński (2003a) \\
\hline $\begin{array}{l}\text { Cladonia furcata (Huds.) Schrad. (ssp. } \\
\text { furcata) }\end{array}$ & soil & also Cieśliński (2003a) \\
\hline Cladonia phyllophora Hoffm. & soil & also Cieśliński (2003a) \\
\hline Cladonia rangiformis Hoffm. & soil & also Cieśliński (2003a) \\
\hline Cladonia subulata (L.) Weber ex F. H. Wigg. & soil & also Cieśliński (2003a) \\
\hline Evernia prunastri (L.) Ach. & $\begin{array}{l}\text { trunk of Alnus glutinosa, Acer } \\
\text { platanoides, Betula pendula, } \\
\text { wooden crosses }\end{array}$ & also Cieśliński (2003a) \\
\hline Flavoparmelia caperata (L.) Hale & trunk of Alnus glutinosa & Cieśliński (2003a) \\
\hline Hyросеnотусе scalaris (Ach.) M. Choisy & $\begin{array}{l}\text { trunk of Alnus glutinosa, Pinus } \\
\text { sylvestris, Betula pendula, } \\
\text { wooden crosses }\end{array}$ & also Cieśliński (2003a) \\
\hline Hypogymnia physodes (L.) Nyl. & $\begin{array}{l}\text { trunk of Alnus glutinosa, Pinus } \\
\text { sylvestris, Acer platanoides, } \\
\text { Betula pendula, Quercus robur, } \\
\text { Juniperus communis, wooden } \\
\text { crosses and bench, decaying } \\
\text { wood }\end{array}$ & also Cieśliński (2003a) \\
\hline Hypogymnia tubulosa (Schaer.) Hav. & trunk of Betula pendula & \\
\hline Imshaugia aleurites (Ach.) S.L.F. Meyer & $\begin{array}{l}\text { trunk of Pinus sylvestris, } \\
\text { wooden crosses, decaying } \\
\text { wood }\end{array}$ & also Cieśliński (2003a) \\
\hline Lecanora albescens (Hoffm.) Branth \& Rostr. & $\begin{array}{l}\text { stones, stone wall, concrete } \\
\text { and stone tombstones }\end{array}$ & \\
\hline Lecanora carpinea (L.) Vain. & trunk of Alnus glutinosa & \\
\hline Lecanora conizaeoides Nyl. ex Cromb. & $\begin{array}{l}\text { trunk of Alnus glutinosa, Pinus } \\
\text { sylvestris, wooden crosses }\end{array}$ & also Cieśliński (2003a) \\
\hline Lecanora dispersa (Pers.) Sommerf. & $\begin{array}{l}\text { stones, stone wall, concrete } \\
\text { and stone tombstones }\end{array}$ & \\
\hline $\begin{array}{l}\text { Lecanora polytropa (Ehrh. ex Hoffm.) } \\
\text { Rabenh. }\end{array}$ & stone wall & \\
\hline
\end{tabular}


Tab. 1 cont.

\begin{tabular}{|c|c|c|}
\hline Lecanora pulicaris (Pers.) Ach. & trunk of Alnus glutinosa & also Cieśliński (2003a) \\
\hline Lecanora varia (Hoffm.) Ach. & wooden crosses & \\
\hline Lecidella elaeochroma (Ach.) M. Choisy & trunk of Alnus glutinosa & \\
\hline Lecidella stigmatea (Ach.) Hertel \& Leuckert & concrete tombstones & \\
\hline Lepraria sp. & $\begin{array}{l}\text { trunk of Acer platanoides, } \\
\text { Alnus glutinosa, Quercur robur, } \\
\text { Pinus sylvestris, Juniperus } \\
\text { communis, wooden crosses, } \\
\text { decaying wood }\end{array}$ & \\
\hline $\begin{array}{l}\text { Melanelixia fuliginosa (Duby) O. Blanco et al. } \\
\text { ssp. glabratula (Lamy) J. R. Laundon }\end{array}$ & $\begin{array}{l}\text { trunk of } \text { Alnus glutinosa, } \\
\text { wooden crosses }\end{array}$ & \\
\hline Parmelia sulcata Taylor & $\begin{array}{l}\text { trunk of Alnus glutinosa, Acer } \\
\text { platanoides, Quercus robur, } \\
\text { Betula pendula, wooden } \\
\text { crosses and bench }\end{array}$ & also Cieśliński (2003a) \\
\hline Parmelina tiliacea (Hoffm.) Hale & wooden crosses & \\
\hline Parmeliopsis ambigua (Wulfen) Nyl. & $\begin{array}{l}\text { trunk of Pinus sylvestris, } \\
\text { wooden crosses }\end{array}$ & also Cieśliński (2003a) \\
\hline Pertusaria amara (Ach.) Nyl. & trunk of Acer platanoides & \\
\hline Phaeophyscia nigricans (Flörke) Moberg & concrete tombstones & \\
\hline Phaeophyscia orbicularis (Neck.) Moberg & stones, stone tombstones & \\
\hline Phlyctis argena (Spreng.) Flot. & $\begin{array}{l}\text { trunk of Acer platanoides, } \\
\text { Alnus glutinosa }\end{array}$ & \\
\hline Physcia adscendens H. Olivier, nom. cons & concrete tombstones & \\
\hline Physcia caesia (Hoffm.) Fürnr. & $\begin{array}{l}\text { stones, stone and concrete } \\
\text { tombstones }\end{array}$ & \\
\hline Physcia dubia (Hoffm.) Lettau & wooden crosses, stone wall & \\
\hline $\begin{array}{l}\text { Placynthiella icmalea (Ach.) Coppins \& P. } \\
\text { James }\end{array}$ & decaying wood & also Cieśliński (2003a) \\
\hline $\begin{array}{l}\text { Platismatia glauca (L.) W.L. Culb. \& C.F. } \\
\text { Culb. }\end{array}$ & $\begin{array}{l}\text { bark of Betula pendula, } \\
\text { wooden crosses }\end{array}$ & \\
\hline $\begin{array}{l}\text { Pleurosticta acetabulum (Neck.) Elix \& } \\
\text { Lumbsch in Lumbsch, Kothe \& Elix }\end{array}$ & trunk of $A$ lnus glutinosa & Cieśliński (2003a) \\
\hline $\begin{array}{l}\text { Porpidia crustulata (Ach.) Hertel \& Knoph } \\
\text { in Hertel }\end{array}$ & stone wall & \\
\hline $\begin{array}{l}\text { Porpidia rugosa (Taylor) Coppins \& Fryday } \\
\text { [=P. glaucophaea (Körb.) Hertel \& Knoph] }\end{array}$ & erratic blocks & Cieśliński (2003a) \\
\hline $\begin{array}{l}\text { Protoparmeliopsis muralis (Schreb.) M. } \\
\text { Choisy }\end{array}$ & $\begin{array}{l}\text { wooden crosses, stone and } \\
\text { concrete tombstones }\end{array}$ & also Cieśliński (2003a) \\
\hline Pseudevernia furfuracea (L.) Zopf & $\begin{array}{l}\text { trunk of Pinus sylvestris, } \\
\text { Juniperus communis, wooden } \\
\text { crosses }\end{array}$ & \\
\hline $\begin{array}{l}\text { Scoliciosporum chlorococcum (Graewe ex } \\
\text { Stenh.) Vězda }\end{array}$ & $\begin{array}{l}\text { trunk of Alnus glutinosa, } \\
\text { Quercus robur, Betula pendula, } \\
\text { wooden crosses }\end{array}$ & also Cieśliński (2003a) \\
\hline Trapeliopsis granulosa (Hoffm.) Lumbsch & soil & also Cieśliński (2003a) \\
\hline Tuckermanopsis chlorophylla (Willd.) Hale & $\begin{array}{l}\text { trunk of Pinus sylvestris Betula } \\
\text { pendula, wooden crosses }\end{array}$ & also Cieśliński (2003a) \\
\hline Usnea filipendula Stirt. & $\begin{array}{l}\text { trunk of Pinus sylvestris, Betula } \\
\text { pendula, wooden crosses }\end{array}$ & \\
\hline Usnea hirta (L.) F. H. Wigg. & $\begin{array}{l}\text { trunk of Pinus sylvestris, Betula } \\
\text { pendula, wooden crosses }\end{array}$ & \\
\hline Verrucaria muralis Ach. & concrete tombstones & \\
\hline Verrucaria nigrescens Pers. & concrete tombstones & \\
\hline Xanthoparmelia conspersa (Ach.) Hale & stones & also Cieśliński (2003a) \\
\hline $\begin{array}{l}\text { Xanthoria polycarpa (Hoffm.) Th. Fr. ex } \\
\text { Rieber }\end{array}$ & $\begin{array}{l}\text { trunk of Juniperus communis, } \\
\text { concrete tombstones, stone } \\
\text { wall }\end{array}$ & also Cieśliński (2003a) \\
\hline
\end{tabular}


erratic block in shaded and moist place. It is a very rare species, growing in very few stations on the Polish lowland.

Epigeic lichens. The terricolous lichens grow in the Holy Hill vicinity in pine tree forests. 10 species of terricolous lichens were identified on the soil, including 7 exclusive species (Cetraria aculeata, C. islandica, Cladonia arbuscula subsp. mitis, C. furcata, C. phyllophora, C. rangiformis). Trapeliopsis granulosa grows on sandy road side - space.

\section{CONCLUSIONS}

There is no data concerning the lichen biota of Holy Grabarka Hill. Several species of lichens from the forests surrounding the Hill have been described by Cieśliński (2003a). Specific character of the Holy Hill, which is situated among forests has inclined to study its lichen biota, and varied stand of trees, wooden crosses, tombstones and stones have suggested that rare and interesting species of lichens can occur there. Despite of a small area, differentiation of habitats and phorophits is so big that 64 species were identifid there. During our own studies the occurence of Flavoparmelia caperata, Pleurosticta acetabulum and Porpidia rugosa, which were mentioned by Cieśliński (2003a), was not confirmed. The lichens form a significant group of organisms in the scenery of the Holy Grabarka Hill. Their presence at all accessible substrata demonstrates positive results of synatropization. One of the distinctive features are wooden crosses overgrown abundantly by lichen thalus.

Acknowledgement. I wish to express my thanks to Reviewer for his precious remarks and advice.

\section{REFERENCENS}

Blanco O., Crespo A., Divakar P. K., Esslinger T. L., Hawksworth D. L., Lumbsch H. T. 2004. Melanelixia and Melanohalea, two new genera segregated from Melanelia (Parmeliaceae) based on molecular and morphological data. Mycol. Res. 108 (8): 873-884.

Bystrek J. 1986. Species of the genus Bryoria Brodo et Hawksw. (Lichenes, Usneaceae) in Europe. Bulletin of the Polish Academy of Sciences, Biol. Ser. 34 (10/12): 293-300.

Bystrek J. 1994. Studien über die Flechtengattungen Usnea in Europa.Wyd. Uniwersytetu Marii CurieSkłodowskiej, Lublin, 69 pp.

Cieśliński S. 2003a. Distribution atlas of lichens (Lichenes) in North-Eastern Poland. Phytocoenosis 15 (N.S.), Suppl. Cartogr. Geobot.15, 430 pp.

Cieśliński 2003b. Red list of extinct and threatened lichenes in Poland. (In:) K. Cżyżewska (ed.). The threat to lichens in Poland. Monogr. Bot. 91: 91-106.

Cieśliński S., Czyżewska K., Fabiszewski J. 2003. Red list of exinct and threatened lichenes in Poland. (In:) K. Czyżewska (ed.). The threat to lichens in Poland. Monogr. Bot. 91: 13-49.

Fałtynowicz W. 2003. The lichens lichenicolous and allied fungi of Poland. An annotated checklist. Szafer Institute of Botany, Polish Academy of Sciences, Kraków, 435 pp.

Kondracki J. 1994. Geografia Polski. Mezoregiony fizyczno-geograficzne. PWN, Warszawa.

Lechowski A. 2004. Między niebem a ziemią. Grabarka. Góra Krzyży (The Hill of Crosses). Wydawnictwa Orthdruk, Białystok.

Radziukiewicz A. 2000. Prawosławie w Polsce. Wydawnictwo Arka, Białystok.

Radziukiewicz A. 2001. Góra krzyży i modlitwy. Wydawnictwo Orthdruk, Białystok.

Dz. U. Nr 168, poz. 1765, Rozporządzenie Ministra Środowiska z dnia 9 lipca 2004 r. w sprawie gatunków dziko występujących grzybów objętych ochroną. 


\section{Porosty Świętej Góry, prawosławnego sanktuarium w Grabarce}

\section{Streszczenie}

W pracy przedstawiono wyniki badań nad porostami Świętej Góry, prawosławnego sanktuarium w Grabarce. Święta Góra Grabarka jest najważniejszym sanktuarium prawosławnym w Polsce. Położona jest w Polsce Północno-Wschodniej, na Podlasiu, między Bugiem a Narwią, na Wysoczyźnie Drohickiej, w południowej części Niziny Północnopodlaskiej.

Święta Góra Grabarka zasłynęła z cudu, jaki miał miejsce na początku 1710 roku podczas epidemii cholery, która dziesiątkowała ludność na terenie Podlasia. Święta Góra Grabarka nazywana jest Górą Krzyży. Nazwa ta wywodzi się od krzyży ofiarnych z epitafiami pisanymi cyrylicą otaczającymi cerkiew Przemienienia Pańskiego. Krzyże stojące wokół niewielkiej, drewnianej cerkwi stały się symbolem Świętej Góry. Dziś na wzgórzu wznosi się około 10 tysięcy krzyży - drewnianych, metalowych, kamiennych, małych i wielkich. Krzyże tradycyjnie przynoszone są przez wiernych od setek lat. Pątnicy stawiają je obok sanktuarium przez cały rok, jednak najwięcej krzyży przybywa w przeddzień Święta Przemienienia Pańskiego (19 sierpnia). Za lasem krzyży znajduje się cmentarz oraz kompleks zabudowań klasztoru świętych Marty i Marii. Żeński klasztor prawosławny powstał w 1947 roku, a w 1957 została wzniesiona cerkiew p. w. Matki Bożej Wspomożycielki i Opiekunki.

Na terenie Świętej Góry Grabarki i jej okolic odnotowano 64 gatunki porostów (Tab. 1), z 35 rodzajów. Najliczniej reprezentowane są rodzaje Cladonia - 8 gatunków, Lecanora 7, Caloplaca -4 . Porosty występują we wszystkich podstawowych formach morfologicznych. Najliczniej reprezentowane są porosty epifityczne. 11 gatunków umieszczonych jest na Czerwonej liście porostów wymarłych i zagrożonych w Polsce (Cieśliński et al. 2003), w tym 3 gatunki w kategorii wymierających - EN (Bryoria crispa, Flavoparmelia caperata, Pleurosticta acetabulum), 6 w kategorii narażonych - VU (Cetraria islandica, Parmelina tiliacea, Porpidia rugosa, Tuckermanopsis chlorophylla, Usnea filipendula, U. hirta), 2 - w kategorii bliskich zagrożeniu - NT (Evernia prunastri, Hypogymnia tubulosa). 